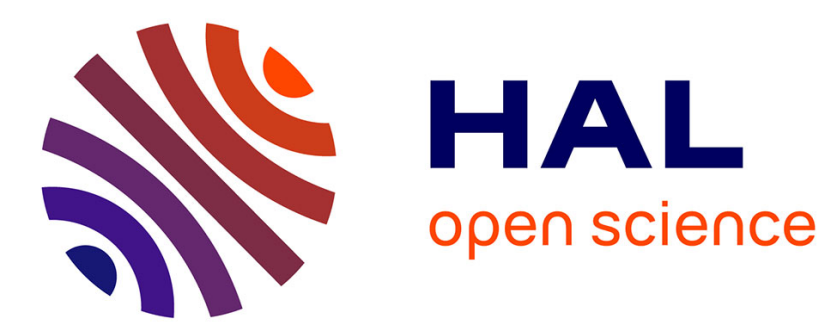

\title{
Comparison of Numerical Methods in the Contrast Imaging Problem in NMR
}

\author{
Bernard Bonnard, Mathieu Claeys, Olivier Cots, Pierre Martinon
}

\section{To cite this version:}

Bernard Bonnard, Mathieu Claeys, Olivier Cots, Pierre Martinon. Comparison of Numerical Methods in the Contrast Imaging Problem in NMR. 52nd IEEE Conference on Decision and Control, Dec 2013, Firenze, Italy. hal-00800436

\section{HAL Id: hal-00800436 https://hal.inria.fr/hal-00800436}

Submitted on 13 Mar 2013

HAL is a multi-disciplinary open access archive for the deposit and dissemination of scientific research documents, whether they are published or not. The documents may come from teaching and research institutions in France or abroad, or from public or private research centers.
L'archive ouverte pluridisciplinaire HAL, est destinée au dépôt et à la diffusion de documents scientifiques de niveau recherche, publiés ou non, émanant des établissements d'enseignement et de recherche français ou étrangers, des laboratoires publics ou privés. 


\title{
COMPARISON OF NUMERICAL METHODS IN THE CONTRAST IMAGING PROBLEM IN NMR
}

\author{
Bernard Bonnard, Mathieu Claeys, Olivier Cots and Pierre Martinon
}

\begin{abstract}
In this article, the contrast imaging problem in nuclear magnetic resonance is modeled as a Mayer problem in optimal control. A first synthesis of locally optimal solutions is given in the single-input case using geometric methods based on Pontryagin's maximum principle. We then compare these results using direct methods and a moment-based approach, and make a first step towards global optimality. Finally, some preliminary results are given in the bi-input case.
\end{abstract}

\section{INTRODUCTION}

A classical problem in Nuclear Magnetic Resonance (NMR) spectroscopy is to control, using a magnetic field, a spin-1/2 particle in a dissipative environment whose dynamics is governed by the Bloch equation

$$
\begin{aligned}
& \frac{\mathrm{d} M_{x}}{\mathrm{~d} \tau}=-M_{x} / T_{2}+\omega_{y} M_{z}-\Delta \omega M_{y} \\
& \frac{\mathrm{d} M_{y}}{\mathrm{~d} \tau}=-M_{y} / T_{2}-\omega_{x} M_{z}+\Delta \omega M_{x} \\
& \frac{\mathrm{d} M_{z}}{\mathrm{~d} \tau}=\left(M_{0}-M_{z}\right) / T_{1}+\omega_{x} M_{y}-\omega_{y} M_{x}
\end{aligned}
$$

where the state variables correspond to the magnetization vector $M=\left(M_{x}, M_{y}, M_{z}\right), T_{1}$ and $T_{2}$ are the relaxation rates, $\Delta \omega$ is the resonance offset and $\tau$ is the time. In this model the control is the magnetic field $\omega=\left(\omega_{x}, \omega_{y}, 0\right)$ which is bounded here by $|\omega| \leq \omega_{\max }=32.3 \mathrm{~Hz}$. In order to set the equilibrium of the free motion to $(0,0,1)$, we normalize the coordinates to $q=(x, y, z)=\left(M_{x}, M_{y}, M_{z}\right) / M_{0}$, and $q$ belongs to the Bloch ball $|q| \leq 1$. We then normalize the control by $u=\omega / \omega_{\max }$ and the normalized time is $t=\tau \omega_{\max }$. In this paper, we analyze the simplified model, $\Delta \omega=0$, where homogeneity of the magnetic fields is assumed and we have the following normalized system

$$
\begin{array}{ll}
\frac{\mathrm{d} x}{\mathrm{~d} t}=-\Gamma x & +u_{2} z \\
\frac{\mathrm{d} y}{\mathrm{~d} t}=-\Gamma y \quad-u_{1} z & \\
\frac{\mathrm{d} z}{\mathrm{~d} t}=\gamma(1-z) & +u_{1} y-u_{2} x
\end{array}
$$

B. Bonnard is with Institut de Mathématiques de Bourgogne, Université de Bourgogne, 9 avenue Alain Savary, 21078 Dijon, France, bernard.bonnardeu-bourgogne. fr.

M. Claeys is with CNRS; LAAS; 7 avenue du colonel Roche, F-31077 Toulouse; France and with Université de Toulouse; UPS, INSA, INP, ISAE; UT1, UTM, LAAS; F-31077 Toulouse; France, mclaeys@laas.fr.

O. Cots is with INRIA Sophia Antipolis Méditerranée, 06902 Sophia Antipolis, France, olivier.cots@inria.fr.

P. Martinon is with Inria and Ecole Polytechnique, 91128 Palaiseau France, martinonecmap.polytechnique.fr. where $\Gamma=1 /\left(\omega_{\max } T_{2}\right)$ and $\gamma=1 /\left(\omega_{\max } T_{1}\right)$. In the contrast problem, we consider two uncoupled spin-1/2 systems corresponding to different particles, each of them solutions of the Bloch equation (2) with respective damping coefficients, $\left(\gamma_{1}, \Gamma_{1}\right)$ and $\left(\gamma_{2}, \Gamma_{2}\right)$ and controlled by the same magnetic field. By denoting each system by $\frac{\mathrm{d} q_{i}}{\mathrm{~d} t}=F_{i}\left(q_{i}, \Lambda_{i}, u\right)$, $\Lambda_{i}=\left(\gamma_{i}, \Gamma_{i}\right)$ and $q_{i}=\left(x_{i}, y_{i}, z_{i}\right)$ the magnetization vector for each spin particle, this leads to consider the system

$$
\frac{\mathrm{d} q_{1}}{\mathrm{~d} t}=F_{1}\left(q_{1}, \Lambda_{1}, u\right), \quad \frac{\mathrm{d} q_{2}}{\mathrm{~d} t}=F_{2}\left(q_{2}, \Lambda_{2}, u\right)
$$

which is written shortly as $\frac{\mathrm{d} x}{\mathrm{~d} t}=F(x, u)$, where $x=\left(q_{1}, q_{2}\right)$ is the state variable.

The contrast problem by saturation is the following optimal control problem $(O C P)$ : starting from the equilibrium point $x_{0}=((0,0,1),(0,0,1))$, reach in a given transfer time $t_{f}$ the final state $q_{1}\left(t_{f}\right)=0$ (corresponding to zero magnetization of the first spin, called saturation) while maximizing $\left|q_{2}\left(t_{f}\right)\right|^{2}$, the contrast being $\left|q_{2}\left(t_{f}\right)\right|$. The contrast problem can be stated as a Mayer problem given by the following smooth conditions:

1) A system $\frac{\mathrm{d} x}{\mathrm{~d} t}=F(x, u), x \in \mathbf{X} \subseteq \mathbb{R}^{n}$, with

$$
\mathbf{X}=\left\{x=\left(q_{1}, q_{2}\right) \in \mathbb{R}^{n}:\left|q_{1}\right| \leq 1,\left|q_{2}\right| \leq 1\right\},
$$

with fixed initial state $x(0)=x_{0}$ and where the control belongs to the control domain $\mathbf{U}=\{u \in \mathbb{R}:|u| \leq 1\}$.

2) A terminal manifold to reach,

$$
\mathbf{X}_{\mathbf{f}}=\left\{x=\left(q_{1}, q_{2}\right) \in \mathbb{R}^{n}: q_{1}=0,\left|q_{2}\right| \leq 1\right\} \subset \mathbf{X},
$$

defined by $f(x)=0$, where $f: \mathbb{R}^{n} \rightarrow \mathbb{R}^{k}$.

3) A cost to be minimized of the form $\min _{u(\cdot)} c\left(x\left(t_{f}\right)\right)$ where $c: \mathbb{R}^{n} \rightarrow \mathbb{R}$ is a regular mapping and $t_{f}$ is the final time.

In practical experiments we consider two cases:

a) The bi-input case where $x=\left(q_{1}, q_{2}\right) \in \mathbb{R}^{6} \cap \mathbf{X}$ and $|u|=\left(u_{1}^{2}+u_{2}^{2}\right)^{1 / 2} \leq 1$.

b) The single-input case where the system is restricted to $x_{1}=x_{2}=0$, the control field is restricted to the real field, i.e., $u_{2}=0$, and each spin is restricted to the plane $q_{i}=\left(y_{i}, z_{i}\right)$.

The use of particular pulse sequences (i.e. control law $u(\cdot))$ in the contrast problem is not new since this question was raised at the beginning of the development of Magnetic Resonance Imaging (MRI) in the seventies. Different strategies based on intuitive reasoning have been proposed such as the Inversion Recovery Sequence. Recently, S. J. Glaser 
introduced the optimal control point of view [10] and analysed the problem in his group using an adapted numerical scheme (the GRAPE algorithm [9]). A different approach based on Pontryagin's Maximum Principle was recently used to select minimizers in the single input case [5]. This leads to a numerical investigation described in [6] using the HAMPATH ${ }^{1}$ software, based on the indirect methods: shooting and differential continuation.

One objective of this article is to compare these results with a direct method implemented with the $\mathrm{BOCOP}^{2}$ toolbox [2]. This approach relies on a time discretization of the state and control variables, using a high order implicit formula. The resulting nonlinear programming problem is then solved by interior point techniques, with automatic computation of the sparse derivatives. Direct methods fall in the classe of local optimization, like indirect approaches, but are typically easier to initialize. Their main drawback is that due to the discretization, solutions tend to be coarser than the ones obtained by indirect methods.

A distinguishing feature of the contrast problem are its many locally optimal solutions, which can be computed by the previously described direct and indirect methods. An important question is then to assert global optimality. The second objective of this paper is to use a moment/Linear Matrix Inequality (LMI) technique [8], [12] to compute such an estimate. In fact, the method allows to build a hierarchy of relaxations of the original problem, that is compute lower bounds on the true cost, each in the form of a convex LMI problem. Because of this convexity, the relaxations can be solved by off-the-shelf software (we used Sedumi [13]) without any expert knowledge besides problem formulation. In particular, no specific inital point needs to be supplied.

The paper is organized in four sections. The first one settles the necessary conditions applied to the contrast problem. The second section details the three numerical methods used in the third and fourth sections, which present the results respectively in the single-input and bi-input cases.

\section{NECESSARY OPTIMALITY CONDITIONS}

In this section we briefly recall the theoretical framework, see [5] for the details.

\section{A. Maximum principle}

Proposition 1. If $u^{*}$ with corresponding trajectory $x^{*}$ is optimal then the following necessary optimality conditions are satisfied. Denoting $H(x, p, u)=\langle p, F(x, u)\rangle$ as the pseudo-Hamiltonian, there exists $p^{*}(\cdot)$ such that for almost every $t \in\left[0, t_{f}\right]$,

(i) $\frac{\mathrm{d} x^{*}}{\mathrm{~d} t}=\frac{\partial H}{\partial p}\left(x^{*}, p^{*}, u^{*}\right), \quad \frac{\mathrm{d} p^{*}}{\mathrm{~d} t}=-\frac{\partial H}{\partial x}\left(x^{*}, p^{*}, u^{*}\right)$

(ii) $H\left(x^{*}, p^{*}, u^{*}\right)=\max _{v \in U} H\left(x^{*}, p^{*}, v\right)$ (maximization condition)

and the following boundary conditions

(iii) $f\left(x^{*}\left(t_{f}\right)\right)=0$

\footnotetext{
${ }^{1}$ http: //cots.perso.math.cnrs.fr/hampath
}

${ }^{2}$ http: //bocop.org (iv) $p^{*}\left(t_{f}\right)=p_{0} \frac{\partial c}{\partial x}\left(x^{*}\left(t_{f}\right)\right)+\sum_{i=1}^{k} \sigma_{i} \frac{\partial f_{i}}{\partial x}\left(x^{*}\left(t_{f}\right)\right), \sigma=$ $\left(\sigma_{1}, \ldots, \sigma_{k}\right) \in \mathbb{R}^{k}, p_{0} \leq 0$ (transversality condition)

Definition 1. The solutions of conditions (i) and (ii) of Prop. 1 are called extremals and BC-extremals if they satisfy the boundary conditions.

\section{B. Application to the contrast problem}

State space: Since the Bloch ball is invariant for the dynamics of each spin particle, the state constraints can be omitted for analysis, and the maximum principle can be applied.

Boundary conditions: In the contrast problem, $x=$ $\left(q_{1}, q_{2}\right), f=0$ is the set $q_{1}=0$, and the cost to minimize is $c(x)=-\left|q_{2}\right|^{2}$. Hence, splitting the adjoint vector into $p=\left(p_{1}, p_{2}\right)$, we deduce from the transversality condition that $p_{2}\left(t_{f}\right)=-2 p_{0} q_{2}\left(t_{f}\right), p_{0} \leq 0$. If $p_{0}$ is nonzero, it can be normalized to $p_{0}=-1 / 2$.

Next, we compute the extremal curves, solutions of the maximum principle.

Bi-input case: The system is written as $\frac{\mathrm{d} x}{\mathrm{~d} t}=F_{0}(x)+$ $u_{1} F_{1}(x)+u_{2} F_{2}(x),|u| \leq 1$ and the maximization condition in Prop. 1 leads to the following parameterization of the extremal controls:

$$
u_{1}=\frac{H_{1}}{\sqrt{H_{1}^{2}+H_{2}^{2}}}, \quad u_{2}=\frac{H_{2}}{\sqrt{H_{1}^{2}+H_{2}^{2}}},
$$

where $H_{i}=\left\langle p, F_{i}(x)\right\rangle$ are Hamiltonian lifts outside the switching surface $\Sigma: H_{1}=H_{2}=0$. Plugging such a $u$ into the pseudo-Hamiltonian gives the true Hamiltonian $H_{n}=H_{0}+\left(H_{1}^{2}+H_{2}^{2}\right)^{1 / 2}$. The smooth solutions of the corresponding vector field are called extremals of order zero.

The single-input case: Consider the case where the control is restricted to a single input and the system is written $\frac{\mathrm{d} x}{\mathrm{~d} t}=F(x)+u G(x)$, where $x$ belongs to a 4-dimensional space $\mathbf{X}$ and $|u| \leq 1$. We denote $H_{F}$ and $H_{G}$ to be the respective Hamiltonian lifts.

Applying the maximization condition, there are two types of extremals.

- Regular extremals: The control is given by $u(t)=$ $\operatorname{sgn} H_{G}(z(t)), z=(x, p)$. If the number of switchings is finite, it is called bang-bang.

- Singular extremals: Since the system is linear in $u$, the maximization condition leads, in the singular case, to the condition $H_{G}(z(t))=0$. The corresponding singular control is

$$
u_{s}=-\frac{\left\{\left\{H_{G}, H_{F}\right\}, H_{F}\right\}}{\left\{\left\{H_{G}, H_{F}\right\}, H_{G}\right\}},
$$

where $\{\cdot, \cdot\}$ is the standard Poisson bracket.

According to the maximum principle, an optimal solution is the concatenation of bang and singular arcs and the complexity of this sequence is measured by the number of concatenated arcs. This leads to the following lemma: 
Lemma 1. In the contrast problem, the simplest BC-extremal is of the form BS, that is a Bang arc followed by a Singular arc.

A straightforward computation gives the following result.

Proposition 2. The extremals of the single-input case are extremals of the bi-input case.

A limit case in the contrast problem is the case where the transfer time $t_{f}$ is exactly the time $T_{\min }$ to transfer the first spin to zero, the optimal control producing a final contrast $\left|q_{2}\left(T_{\min }\right)\right|^{2}$. We have the following proposition

Proposition 3. The time-minimal solution of the first spin system can be embedded as an extremal solution of the contrast problem with $p_{0}=0$ in the transversality condition of Prop. 1.

\section{THE NUMERICAL METHODS}

In this section we present the three numerical methods used for the resolution of the contrast problem.

\section{$A$. HAMPATH}

The HAMPATH software is described in details in [6]. It is based upon indirect methods: shooting, differential continuation methods, and computation of the solutions of the variational equations. We give in this section a summary of the principles used to solve the contrast problem in the single-input case, excerpted from [6], [4]. In this case, the geometrical study of the contrast problem ensures that the optimal solution is a concatenation of bang and singular arcs. We note nBS a structure composed by $\mathrm{n}$ Bang-Singular sequences.

The strategy is to first regularize the problem in order to detect the BS-sequences and find a good approximation of the solution. We use the following regularization which transforms the Mayer problem in the Bolza form with cost:

$$
c\left(x\left(t_{f}\right)\right)+(1-\lambda) \int_{0}^{t_{f}}|u|^{2-\lambda} \mathrm{d} t, \quad \lambda \in[0,1],
$$

with fixed final time $t_{f}$. The regularized Hamiltonian is

$H(x, p, \lambda)=p^{0}(1-\lambda)|u(\cdot)|^{2-\lambda}+H_{F}(x, p)+u(\cdot) H_{G}(x, p)$,

where $u(\cdot)$ stands for $u(x, p, \lambda)$ and

$$
u(x, p, \lambda)=\operatorname{sgn}\left(H_{G}(x, p)\right)\left(\frac{2\left|H_{G}(x, p)\right|}{(2-\lambda)(1-\lambda)}\right)^{\frac{1}{1-\lambda}} .
$$

The homotopic function $h: \Omega \subset \mathbb{R}^{n} \times[0,1) \rightarrow \mathbb{R}^{n}$ given by the transversality conditions is

$$
h\left(p_{0}, \lambda\right)=\left(\begin{array}{c}
q_{1}\left(t_{f}, x_{0}, p_{0}, \lambda\right) \\
q_{2}\left(t_{f}, x_{0}, p_{0}, \lambda\right)-p_{2}\left(t_{f}, x_{0}, p_{0}, \lambda\right)
\end{array}\right),
$$

with $x_{0}=((0,1),(0,1))$. We first solve $h\left(p_{0}, \lambda\right)_{\mid \lambda=0}=0$ and then use differential continuation to get the initial adjoint vector for $\lambda=\lambda_{f}=1-\varepsilon$, with a small $\varepsilon>0$. We use the solution at $\lambda_{f}$ as an initial condition for the resolution of the contrast problem, i.e. at $\lambda=1$, by multiple shooting method. Up to this point, the transfer duration $t_{f}$ is fixed to an arbitrary value. Finally, we use a differential continuation technique in order to to study the behavior of the solutions regarding to the parameter $t_{f}$.

An important issue in the contrast problem is to deal with the many local solutions, such that, for a given value $\overline{t_{f}}$ of the parameter, we must compare the cost associated to each component of $\{h=0\} \cap\left\{t_{f}=\overline{t_{f}}\right\}$, where each branch of $\{h=0\}$ is called a path of zeros. This global aspect is responsible for a possible loss of regularity on the value function $t_{f} \mapsto c\left(x\left(t_{f}\right)\right)$ and on the globally optimal path of zeros.

An important remark is that these methods provide at best local optimal solutions. To ensure the local optimality we can check, in the regular case and for BS structure, sufficient optimality conditions of order two. These can be computed quite easily. On the other hand, it is a very difficult task to ensure global optimality.

\section{B. BOCOP}

The so-called direct approach transforms the infinite dimensional optimal control problem $(O C P)$ into a finite dimensional optimization problem $(N L P)$. This is done by a discretization in time applied to the state and control variables, as well as the dynamics equation. These methods are usually less precise than indirect methods based on Pontryagin's Maximum Principle, but more robust with respect to the initialization. Also, they are more straightforward to apply, hence their wide use in industrial applications.

Summary of the time discretization:

$$
\begin{array}{ll}
t \in\left[0, t_{f}\right] & \rightarrow\left\{t_{0}=0, \ldots, t_{N}=t_{f}\right\} \\
z(\cdot), u(\cdot) & \rightarrow X=\left\{z_{0}, \ldots, z_{N}, u_{0}, \ldots, u_{N-1}, t_{f}\right\} \\
\hline \text { Criterion } \rightarrow \min c\left(z_{N}\right) \\
\text { Dynamics } \rightarrow(\text { ex: Euler }) z_{i+i}=z_{i}+h f\left(z_{i}, u_{i}\right) \\
\text { Adm. Cont. } \rightarrow-1 \leq u_{i} \leq 1 \\
\text { Bnd. Cond. } \rightarrow \Phi\left(z_{0}, z_{N}\right)=0
\end{array}
$$

We therefore obtain a nonlinear programming problem on the discretized state and control variables

$$
(N L P)\left\{\begin{array}{l}
\min F(z)=c\left(z_{N}\right) \\
L B \leq C(z) \leq U B
\end{array}\right.
$$

All tests were run using the BoCOP software [2]. The discretized nonlinear optimization problem is solved by the well-known IPOPT solver [14] with MUMPS [1], while the derivatives are computed by sparse automatic differentiation with ADOL-C [15] and COLPACK [7].

\section{LMI}

The moment approach is a global optimization technique that transforms a non-linear, possibly infinite-dimensional optimization problem into convex, finite-dimensional relaxations in the form of Linear Matrix Inequalities (LMI). We follow [12] for the specific case of optimal control with bounded controls and [11] for the main steps of the method. 
The first step is to embed problem $(O C P)$ into a Linear Program $(L P)$ on measures, by the use of so called occupation measures encoding admissible trajectories. For each admissible control $u(t)$, define its corresponding time occupation measure $\mu[u(t)] \in \mathcal{M}^{+}\left(\mathbf{K}:=\left[0, t_{f}\right] \times \mathbf{U} \times \mathbf{X}\right)$, a positive Radon measure, as:

$$
\mu[u(t)](\mathbf{A}, \mathbf{B}, \mathbf{C}):=\int_{\left[0, t_{f}\right] \cap \mathbf{A}} \delta_{u(t)}(\mathbf{B}) \delta_{x[u(t)](t)}(\mathbf{C}) d t .
$$

Here, $\delta_{x^{*}}$ is the Dirac measure located at $x^{*}$, and $\mathbf{A}, \mathbf{B}$ and $\mathbf{C}$ are Borel subsets of resp. $\left[0, t_{f}\right], \mathbf{U}$ and $\mathbf{X}$. That is, $\mu[u(t)]$ measures the time "spent" by the admissible triplet $(t, u(t), x[u(t)](t))$ on Borel subsets of K. Similarly, we define the final state occupation measure $\mu_{f}[u(t)] \in$ $\mathcal{M}^{+}\left(\mathbf{X}_{\mathbf{f}}\right)$ for the same admissible control as:

$$
\mu_{f}[u(t)](\mathbf{C}):=\delta_{x[u(t)]\left(t_{f}\right)}(\mathbf{C}) .
$$

Proposition 4 (Measure embedding). Control problem $(O C P)$ can be reformulated equivalently in terms of occupation measures:

$$
J_{\mu}=\inf _{\mu[u(t)], \mu_{f}[u(t)]}<c(\cdot), \mu_{f}[u(t)]>
$$

such that, $\forall v(t, x) \in \mathcal{C}^{1}\left(\left[0, t_{f}\right] \times \mathbf{X}\right)$,

$<v\left(t_{f}, \cdot\right), \mu_{f}[u(t)]>-v\left(0, x_{0}\right)=<\frac{\partial v}{\partial t}+\frac{\partial v}{\partial x} \cdot F, \mu[u(t)]>$.

That is, $J_{\mu}=J_{\mathrm{OCP}}$ the original criterion of $(O C P)$.

Consider the following relaxation of problem (4)-(5), where the decision variables are now any measure $\mu \in$ $\mathcal{M}^{+}(\mathbf{K})$ and $\mu_{f} \in \mathcal{M}^{+}\left(\mathbf{X}_{\mathbf{f}}\right)$ instead of occupation measures generated by admissible controls:

$$
J_{\mathrm{LP}}=\inf _{\mu, \mu_{f}}<c(\cdot), \mu_{T}>
$$

such that, $\forall v(t, x) \in \mathcal{C}^{1}\left(\left[0, t_{f}\right] \times \mathbf{X}\right)$,

$$
<v\left(t_{f}, \cdot\right), \mu_{f}>-v\left(0, x_{0}\right)=<\frac{\partial v}{\partial t}+\frac{\partial v}{\partial x} \cdot F, \mu>.
$$

In ill-posed problems, there could be a strict gap induced by the relaxation, i.e. $J_{\mathrm{LP}}<J_{\mu}$, but for the problem at hand, we have the following result:

Proposition 5 (No relaxation gap).

$$
J_{\mathrm{LP}}=J_{\mu}
$$

Proof. The set of admissible vector fields for dynamics $F$ is convex for any $x \in \mathbf{X}$, such that theorem 3.6 (ii) of [12] holds.

Unfortunately, there is no generic tractable method to solve LP problem (6)-(7), and additional structure on problem data is required. For optimal control problem $(O C P)$, this structure is provided by the polynomial cost and dynamics, as well as the basic semi-algebraic characterization of the compact sets $\mathbf{X}$ and $\mathbf{X}_{\mathbf{f}}$. It is then possible to manipulate measures by their moments in a given polynomial basis, which yields a Semi-Definite Program $(S D P)$ on countably many moments, with cost $J_{\mathrm{SDP}}=J_{\mathrm{LP}}$. Truncation of those moment sequences up to degree $2 d$ yields the order $d$ relaxation in the form of a tractable LMI problem, with cost $J_{\text {LMI }}^{d}$. These LMI relaxations yield tighter lower bounds on the true cost as relaxation order is increased, converging monotonically to the solution of $(O C P)$, i.e

Proposition 6 (Monotone convergence).

$$
J_{\mathrm{LMI}}^{n} \uparrow J_{\mathrm{SDP}}=J_{\mathrm{LP}}=J_{\mu}=J_{\mathrm{OCP}}, \quad n \rightarrow \infty
$$

Note that the passage to a given LMI relaxation starting from measure problem (6)-(7) can be fully automated using the GloptiPoly toolbox. To ease exposition, we refer to [8] as well as the toolbox manual for the details of the LMI formulation.

The strong feature of the method is that those LMIs generate lower bounds on the true cost, and can therefore be used as certificates of global optimality. On the other hand, the weak points of the method are its poor algorithmic complexity for unstructured problem, as well as for the special case of optimal control, the unavailability of a generic method to recover controls.

\section{NUMERICAL RESULTS, SINGLE-INPUT CASE}

We present here the results about the single-input case. From the experimental point of view we are interested in the following cases, the parameters being the relaxation times given in seconds.

\section{a) Fluid case.}

Spin 1: Cerebrospinal fluid: $T_{1}=2, T_{2}=0.2$;

Spin 2: Water: $T_{1}=2.5=T_{2}$.

\section{b) Blood case.}

Spin 1: Deoxygenated blood: $T_{1}=1.35, T_{2}=0.05$;

Spin 2: Oxygenated blood: $T_{1}=1.35, T_{2}=0.2$.

\section{A. HamPath}

The results presented in the following part are excerpted from [6]. We give the synthesis of locally optimal solutions obtained in the blood and fluid cases. For the blood, we show on Fig. 1 (left) the contrast for five different components of $\{h=0\}$, for final times $t_{f} \in[1,2] T_{\min }$. The three black branches are made only of BS solutions while the two others are made of $2 \mathrm{BS}$ and $3 \mathrm{BS}$ solutions. For maximizing the contrast, the best policy, drawn as solid lines, is: BS for $t_{f} \in(1,1.294) T_{\min }$ and $3 \mathrm{BS}$ for $t_{f} \in(1.294,2] T_{\min }$. In the special case $t_{f}=T_{\min }$, the solution is $2 \mathrm{BS}$. For the fluid, on Fig. 1 (right), we represent four different branches with 2BS and 3BS solutions. The two greatest values functions intersect around $t_{f}=1.035 T_{\min }$ and the best policy (solid lines) switches between $2 \mathrm{BS}$ and 3BS strategies.

We now compare these results with the direct and LMI methods, in order to assess the optimality of these two suboptimal synthesis.

\section{B. BOCOP}

We present here the results for the direct approach. The only a priori information is the value of the minimum time transfer from [6], used to set the final time in the 

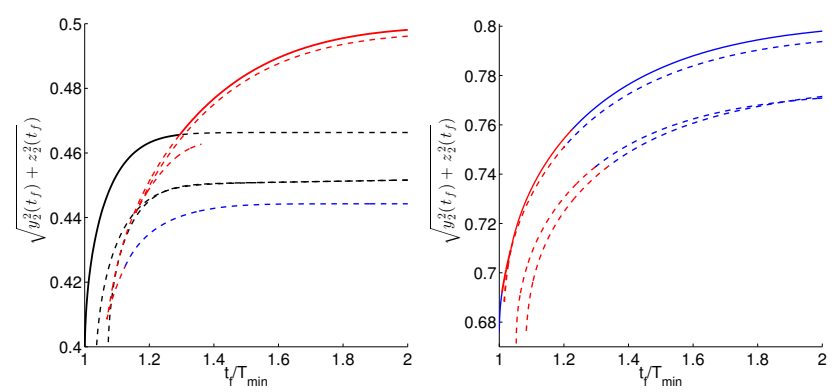

Fig. 1: Hampath. Contrast for various $t_{f}$. Best policy drawn as solid lines, local solutions as dashed lines.

$\left[T_{\min }, 2 T_{\min }\right]$ range. The state and control variables are initialized as constant functions, with the values $y_{1}(\cdot)=0$, $z_{1}(\cdot)=0.5, y_{2}(\cdot)=0, z_{2}(\cdot)=1$, and $u_{x}(\cdot)=0.1$. Each optimization uses this same initial point, and there is no continuation applied here. The discretization methods used are 4th order Gauss or 6th order Lobatto, with 500 to 1000 time steps depending on the problem.

Overall comparison. We show on Fig. 2 the solutions found with BOCOP plotted over the branches identified with HАMPATH in [6]. In most cases the direct solutions belong to one of the already found branches, although some additional branches seem to appear as well. This confirms the complex structure of the extremals for this problem, with several families of local solutions. However, no new solutions with a better contrast were found, which suggests the practical validity of the continuation strategy used with HAMPATH.
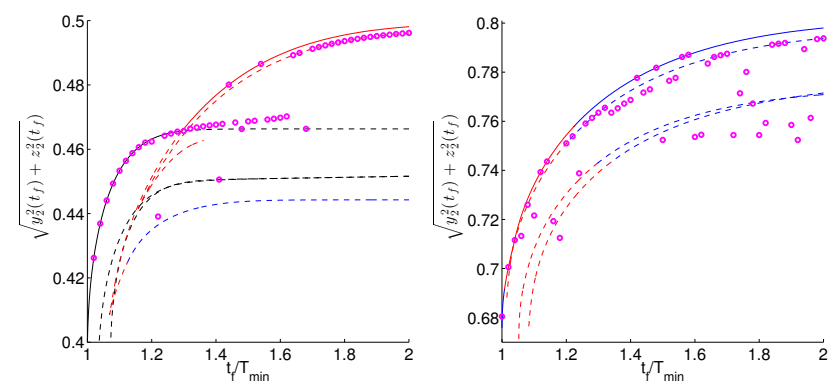

Fig. 2: Bocop and HAMPATH: Blood and Fluid cases.

Blood case. Depending on the final time, we find solutions with either structure BS or 3BS. For small values of $t_{f}$, BoCOP converges to the optimal solution found by HAMPATH. Above $1.3 T_{\min }$, however, BoCOP stays with the BS solution altough we know that the 3BS solutions give a better contrast. Finally, around $1.6 T_{\min }$, BOCOP switches to 3BS solutions, but usually the slighlty worse one of the two (dashed branch on Fig. 2).

Fig. 3 shows both the BOCOP and HAMPATH solutions for $t_{f}=1.1 T_{\min }$. The trajectories for the two spins are identical, and the control is the same, with the exception of some oscillations at the end of the direct solution. These oscillations actually average the "correct" control, which can happen when the system is linear in the control.
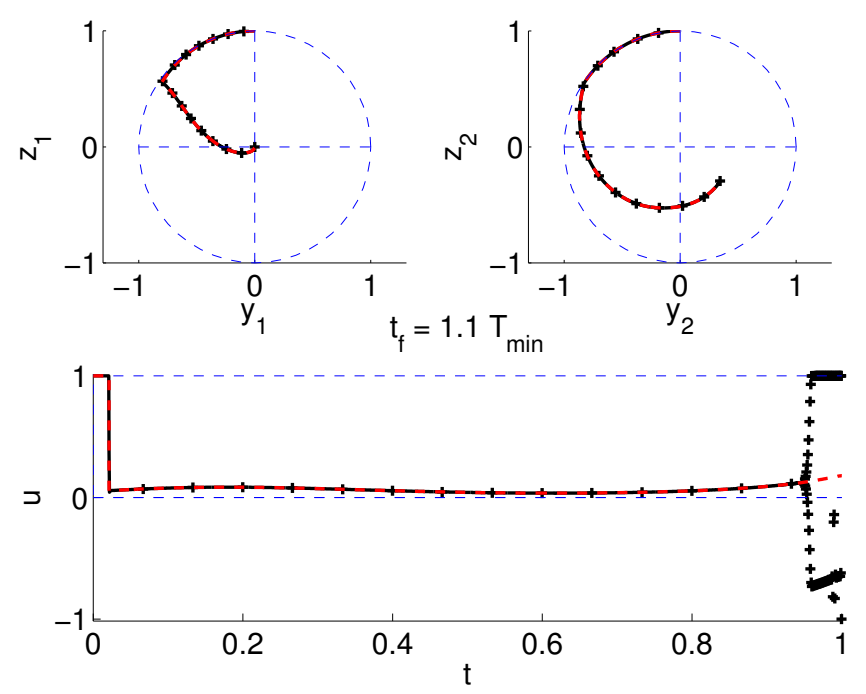

Fig. 3: BoCOP and HAMPATH: Blood case, $t_{f}=1.1 T_{\min }$

Fluid case. The situation in the fluid case is a bit more complicated, and BOCOP converges rather randomly to solutions on different branches, either BS or 3BS. We show on Fig. 4 the solutions for $t_{f}=1.5 T_{\min }$, where BocOP actually finds the best known structure. We observe that both solutions are extremely close, save for a few isolated spikes in the control at the switching times.
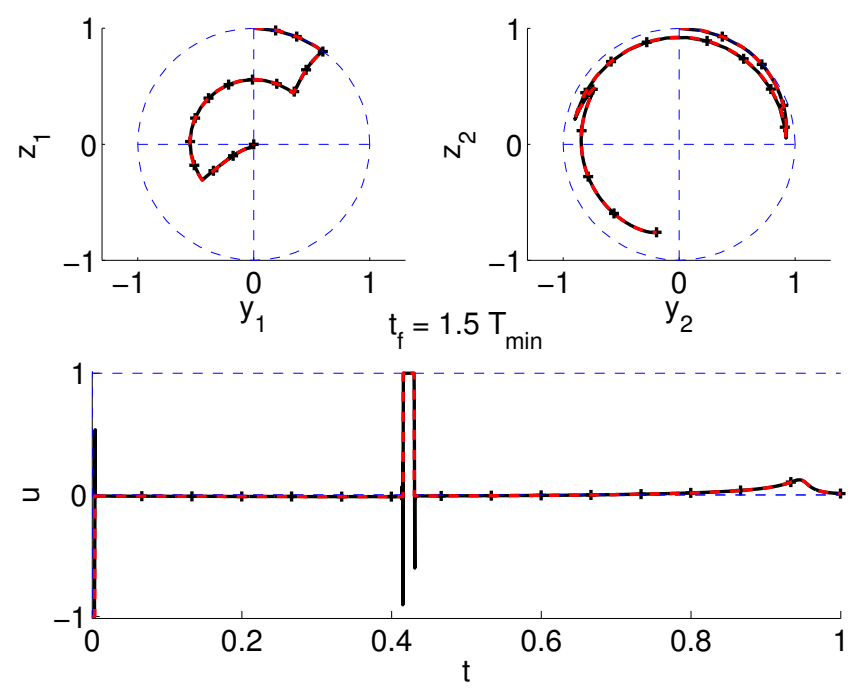

Fig. 4: BocOP and HAMPATH: Fluid case, $t_{f}=1.5 T_{\min }$

Initializing HAMPATH from Bocop. We pick now one case where BOCOP converges to the best solution known, and try to use this data to initialize a shooting in Hampath. More precisely, we recover the control structure and the switching times $t_{i}$ for the bang/singular arcs, as well as $x\left(t_{i}\right), p\left(t_{i}\right)$. We recall that the adjoint states correspond to the multipliers for the discretized dynamics equations in $(N L P)$. With this initialization, we managed to obtain the convergence for the shooting method in HAMPATH, without resorting to continuation techniques. Table I compares the solutions 
from both methods in the blood case, for $T_{f}=1.1 T_{\min }$ and $T_{f}=1.54 T_{\min }$. We see that the constrast and switching times are extremely close. We also compute the relative difference between the renormalized adjoint vector $\mathrm{p}(0)$ and the corresponding multipliers. This difference is as low as a few percent for half the components, and does not exceed $50 \%$ in the worst case. Using higher order discretization formulas and/or more discretization steps may give an even better approximation of $p(0)$.

TABLE I: BOCOP and HAMPATH comparison: Blood case.

\begin{tabular}{|l|l|r|r|}
\hline$t_{f}$ & Method & Contrast & Switching times $t_{i} / t_{f}$ \\
\hline $1.1 T_{\min }$ & HAMPATH & 0.453 & 0.0211 \\
& BOCOP & 0.453 & 0.02 \\
\hline $1.54 T_{\min }$ & HAMPATH & 0.487 & $(0.005,0.348,0.395,0.814,0.855)$ \\
& BOCOP & 0.487 & $(0.004,0.349,0.394,0.815,0.853)$ \\
\hline
\end{tabular}

\begin{tabular}{|l|r|}
\hline$t_{f}$ & $\Delta p(0)$ between BOCOP and HAMPATH \\
\hline $1.1 T_{\min }$ & $(9.81 \%, 3.45 \%, 33.75 \%, 0.61 \%)$ \\
\hline $1.54 T_{\min }$ & $(1.1 \%, 49.37 \%, 29.62 \%, 1.68 \%)$ \\
\hline
\end{tabular}

\section{LMI}

We apply now the LMI method to the contrast problem, in order to obtain upper bounds on the true contrast. Comparing these bounds to the contrast of our solutions then gives an insight about their global optimality.

Table II shows the evolution of the upper bound on the contrast in function of LMI relaxation for the blood case with $t_{f}=T_{\min }$. The first relaxation gives the trivial upper bound, while higher orders yield a monotonically non-increasing sequence of sharper bounds, as expected. Relaxations of orders 5 and 6 yield very similar bounds, but this should not be interpreted as a termination criterion for the LMI method. Table II also shows the evolution of decision variables involved in each LMI relaxation (before any eventual substitution) and the computational load. For all practical purposes, further results from the LMI method were limited to the fifth relaxation given the prohibitive computational load of the sixth one.

TABLE II: LMI: Upper bound on contrast $\sqrt{-J_{\text {LMI }}^{d}}$, LMI variables $N_{d}$ and computation time with respect to the LMI relaxation order $d$ (Blood case)

\begin{tabular}{r|r|r|r}
$d$ & $\sqrt{-J_{\text {LMI }}^{d}}$ & $N_{d}$ & CPU time (s) \\
\hline 1 & 1.0000 & 49 & 1 \\
2 & 0.6092 & 336 & 2 \\
3 & 0.5877 & 1386 & 9 \\
4 & 0.5400 & 4290 & 265 \\
5 & 0.4577 & 11011 & 5147 \\
6 & 0.4442 & 24752 & 63613
\end{tabular}

Fig. 5 compares the evolution of the upper bounds for different values of $t_{f} \in\left[T_{\min }, 2 T_{\min }\right]$ with the best solutions found by HАмРAтH. Also represented is the relative gap between the methods defined as $\left(C_{L M I}-C_{H}\right) / C_{H}$, where $C_{L M I}$ is the LMI upper bound and $C_{H}$ is the contrast found with HAMPATH. At the fifth relaxation, the average gap is $11 \%$, which given the application is satisfactory on the experimental level. Fig. 6 shows the same results for the fluid case. Here, the relative gap on the contrast is about $1 \%$ at the fifth relaxation, which strongly suggest that the solution is actually a global optimum.
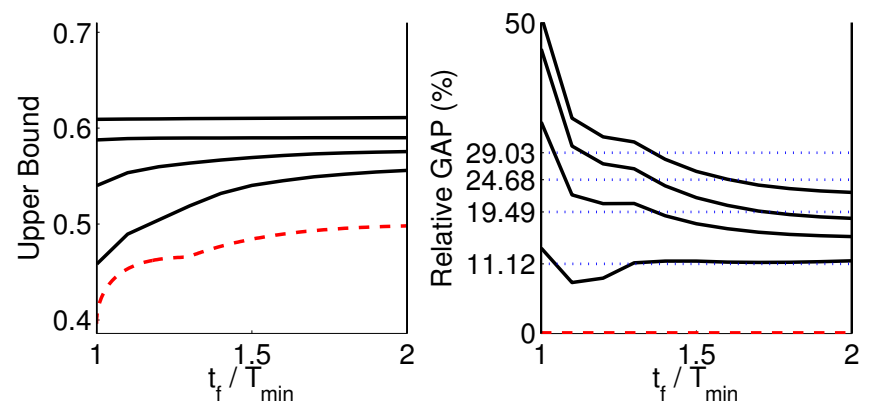

Fig. 5: LMI: Blood case, relaxations 2 to 5
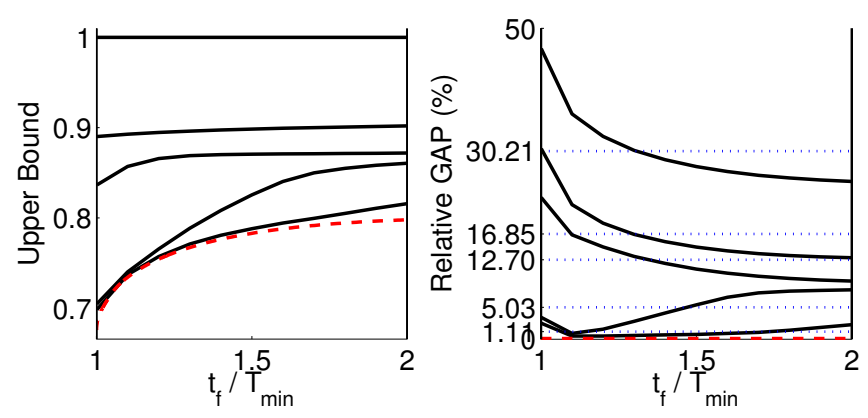

Fig. 6: LMI: Fluid case, relaxations 1 to 5

\section{NUMERICAL RESULTS, BI-INPUT CASE}

We now move on to the bi-input case, with 2 controls and 6 state variables. Here, it is difficult to apply from scratch the continuation strategy with HAMPATH. Therefore, our aim is to try first to obtain some solutions with BOCOP, and use them as a bootstrap for HAMPATH. The LMI method also faces high computational costs, as the problem involves now 7 measured variables (time, 4 states and 2 controls). The 3 rd relaxation is the limit on current machines, and for this low relaxation orders, the bounds are essentially the same as for the single entry case.

We know that single-input extremals are also extremals for the bi-input case. One important question is whether there exist better extremals specific to the bi-input case. According to our first simulations, in the blood case we actually found only single-input type extremals, as shown on Fig. 7, which is identical to the one presented in Fig. 3. On the other hand, in the fluid case, we obtained a bi-input extremal with a slightly better contrast than the single-input solution, see Fig. 8.

\section{CONCLUSION}

The direct method is a very interesting alternative to the regularization procedure (see II-A) in the contrast problem. 

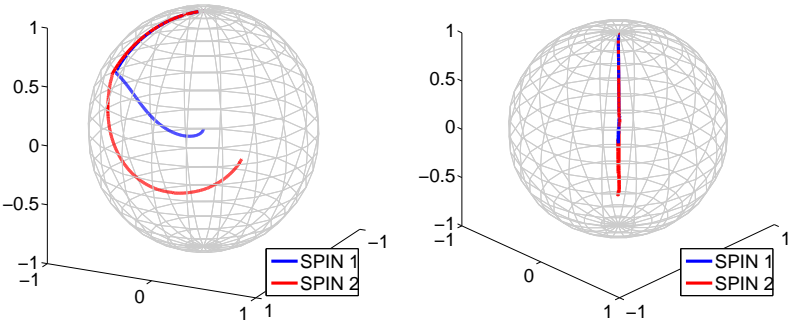

Fig. 7: Bocop: Bi-input case, Blood, $t_{f}=1.1 T_{\min }$

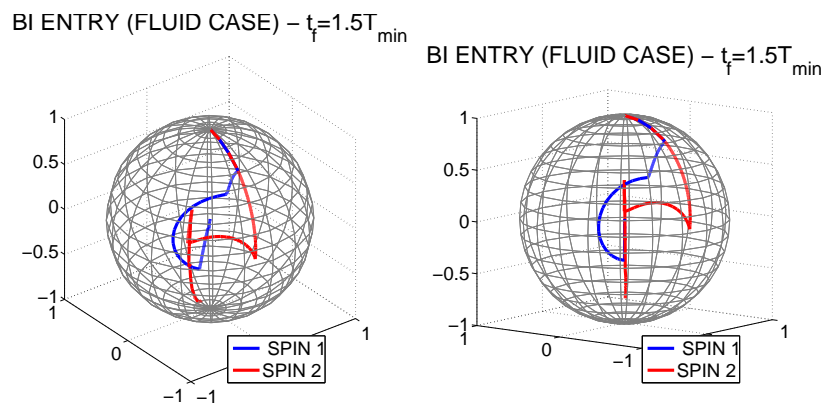

Fig. 8: Bocop: Bi-input case, Fluid, $t_{f}=1.5 T_{\min }$

Despite the many local optima, Bocop allows to get solutions close to the global optimum (see III-B Fig. 2) in a more straightforward way than with HAMPATH, which needs the a priori knowledge of the control structure. This makes its preferable for all practical interests. However, BOCOP gives approximations of solutions which can be refined with HAMPATH (see III-B Table I) and could be used to initialize continuation with respect to the final time in order to get the best possible policy as in III-A Fig. 1.

The LMI techniques allows in the single-input case to get an estimate of the global optimum in the contrast problem which confirms the optimal results obtained previously using the indirect methods [6] (see the details in III-C).

The direct method allows to confirm the gap between the bi-input and the single-input cases. Theorical computations are necessary to understand this phenomenon (see IV for a complete discussion).

\section{REFERENCES}

[1] P. R. Amestoy, I. S. Duff, J. Koster \& J.-Y. L Excellent. A fully asynchronous multifrontal solver using distributed dynamic scheduling, SIAM Journal of Matrix Analysis and Applications, 23 (2001), no. $1,15-41$.

[2] F. J. Bonnans, P. Martinon \& V. Grélard. Bocop - A collection of examples, Technical report, INRIA, 2012. RR-8053.

[3] B. Bonnard, M. Chyba \& J. Mariott, Singular trajectories and the contrast imaging problem in nuclear magnetic resonance, SIAM J. Control Optim., to appear, (2013).

[4] B. Bonnard \& O. Cots, Geometric numerical methods and results in the control imaging problem in nuclear magnetic resonance, Math. Models Methods Appl. Sci., to appear, (2012).

[5] B. Bonnard, O. Cots, S. Glaser, M. Lapert, D. Sugny \& Y. Zhang, Geometric optimal control of the contrast imaging problem in nuclear magnetic resonance, IEEE Trans. Automat. Control, 57 (2012), no. 8, 1957-1969.
[6] O. Cots, Contrôle optimal géométrique : méthodes homotopiques et applications. Phd thesis, http://cots.perso.math.cnrs.fr, Institut Mathématiques de Bourgogne, Dijon, France, 2012.

[7] A. Gebremedhin, A. Pothen \& A. Walther. Exploiting sparsity in jacobian computation via coloring and automatic differentiation: a case study in a simulated moving bed process, In C. Bischof et al, editor, Lecture Notes in Computational Science and Engineering 64, (2008), 327-338, Springer. Proceedings of the Fifth International Conference on Automatic Differentiation (AD2008).

[8] D. Henrion, J. B. Lasserre \& J. Löfberg, GloptiPoly 3: Moments, Optimization and Semidefinite Programming, Optim. Methods and Software, 24 (2009), no. 4-5, 761-779.

[9] N. Khaneja, T. Reiss, C. Kehlet, T. Schulte-Herbrüggen \& S. J. Glaser, Optimal control of coupled spin dynamics: design of NMR pulse sequences by gradient ascent algorithms, J. of Magn. Reson., 172 (2005), 296-305.

[10] M. Lapert, Y. Zhang, M. Janich, S. J. Glaser \& D. Sugny, Exploring the physical limits of saturation contrast in Magnetic Resonance Imaging Sci. Rep. 2, 589 (2012).

[11] J. B. Lasserre, Positive polynomials and their applications, Imperial College Press, London, 2009.

[12] J. B. Lasserre, D. Henrion, C. Prieur \& E. Trélat, Nonlinear optimal control via occupation measures and LMI relaxations, SIAM J. Control Opt., 47 (2008), no. 4, 1643-1666.

[13] J.F. Sturm, Using SeDuMi 1.02, a MATLAB toolbox for optimization over symmetric cones, Optimization Methods and Software 1112 (1999), 625-653. Special issue on Interior Point Methods (CD supplement with software).

[14] A. Wächter \& L.T. Biegler. On the implementation of a primal-dual interior point filter line search algorithm for large-scale nonlinear programming, Mathematical Programming, 106 (2006), no. 1, 25-57.

[15] A. Walther \& A. Griewank. Getting started with adol-c, In U. Naumann and O. Schenk, editors, Combinatorial Scientific Computing. Chapman-Hall CRC Computational Science, (2012). 